\title{
P1-04 Pathologies psychiatriques
}

\author{
C) Springer-Verlag France 2012
}

\author{
P1-04.40 \\ Mémoire involontaire et indicage musical \\ dans la maladie d'Alzheimer \\ J.-C. Broutart ${ }^{1}$, D. Balas ${ }^{2}$, J.-P. Elbaz ${ }^{3}$ \\ ${ }^{1}$ Igam \\ ${ }^{2}$ Université, Nice, France \\ ${ }^{3}$ Fondation GSF-Jean-Louis-Noisiez, Biot, France
}

Introduction : Ce travail est la poursuite de l'étude de la mémoire involontaire et de ses conséquences chez la personne Alzheimer (Les cahiers de l'année gérontologique - volume $2, \mathrm{n}^{\circ} 3$ - septembre 2010). Nous avions montré qu'une musique connue mais inattendue peut éveiller des souvenirs jusqu'alors perdus ; cinq constantes ont été notées : 1) apathie initiale ; 2) déclenchement d'une joie manifestant l'efficacité d'un indice musical ; 3) cascade mémorielle, musicale, sémantique et autobiographique revécue ; 4) bonheur de communiquer ; 5) aucune nostalgie ni tristesse.

Objectifs : Par des séances répétées et espacées de quelques jours : 1) confirmer les résultats précédents ; 2) rechercher si un même indice musical reproduit à chaque séance les mêmes manifestations de mémoire involontaire et récupère les mêmes souvenirs autobiographiques.

Méthodes : L'étude comporte 20 séances d'une heure chacune sur cinq patients Alzheimer avec quatre séances en moyenne par patient. Chaque séance est séparée de la précédente d'un à huit jours. Les séances sont individuelles, entièrement filmées en présence d'un psychologue et d'un personnel soignant. Les indices musicaux efficaces sont d'emblée réutilisés. Les manifestations de joie sont notées, et les souvenirs déclaratifs sont écoutés et analysés.

Résultats : Les constantes suivantes ont été notées : 1) confirmation des résultats de l'étude précédente ; 2) même joie pour même indice musical ; 3) même souvenir autobiographique pour un même indice musical ; 4) enrichissement progressif des souvenirs autobiographiques; 5) restructuration identitaire : fierté, dignité ; 6) mémoire musicale quasi inaltérée quel que soit le stade de l'évolution Alzheimer ; 7) peu ou pas d'action sur la mémoire antérograde.

Conclusion : Les souvenirs sont présents et restitués par les circuits d'une mémoire involontaire. La répétition des séances enrichit la récupération des souvenirs autobiographiques et intensifie le sentiment rassurant de "se retrouver ». Il existe une réciprocité féconde entre mémoire involontaire et maladie d'Alzheimer : 1) la maladie d'Alzheimer par son amnésie antérograde permet la reproductibilité de la mémoire involontaire et donc son étude scientifique ; 2) la mémoire involontaire permet d'assurer quotidiennement aux patients Alzheimer la joie de revivre des souvenirs perdus et le bonheur de les faire partager.

Conflit d'intérêt : les auteurs déclarent ne pas avoir de conflit d'intérêt.

\author{
P1-04.41 \\ Quand la génération Woodstock vieillit : \\ la question des addictions chez les sujets âgés \\ J. Cholet, J.-L. Venisse, G. Berrut \\ CHU de Nantes, Nantes, France
}

Introduction : Avec le vieillissement de la population, les médecins ont dû s'adapter au vieillissement de leurs patients. Ainsi, si de nouvelles pathologies et de nouveaux symptômes ont émergé, comme l'exemple des symptômes psychocomportementaux de la maladie d'Alzheimer, les patients ont aussi vieilli avec leurs propres maladies. Dans le champ des addictions, seule la dépendance aux benzodiazépines semble être une préoccupation des prescripteurs et des cliniciens. Cependant, la génération Woodstock vieillit et la prévalence des sujets âgés présentant une dépendance à une substance psychoactive (SPA), autre que les benzodiazépines, va naturellement s'accroître.

Objectifs : À partir de deux cas cliniques, nous évoquerons les spécificités cliniques et thérapeutiques dans la prise en charge de sujets âgés présentant une dépendance à une SPA.

Méthodes : Deux patients âgés de plus de 60 ans et présentant, l'un, une dépendance à l'alcool et, l'autre, une dépendance aux opiacés sont suivis dans le service. À partir de leurs histoires de vie et de leurs antécédents addictologiques, nous discuterons des spécificités cliniques, au regard des troubles cognitifs associés, et des modalités thérapeutiques (ambulatoires et hospitalières) mises en œuvre.

Résultats : Ces deux patients ont des profils psychopathologiques similaires (début des consommations à l'âge adulte, antécédents de polyconsommations, antécédents de soins spécialisés en addictologie). La coconstruction d'un projet de soin personnalisé et adapté aux ressources du patient prend en compte les capacités d'adaptation, la motivation au changement et les éventuels troubles cognitifs sousjacents des patients. Ainsi, ces deux patients bénéficient d'une prise en charge très différente (postcure hospitalière pour l'un et traitement de substitution orale en ambulatoire pour l'autre).

Conclusion : De par leur impact sur le fonctionnement global et la qualité de vie des patients, les conduites addictives du sujet âgé nécessitent que le clinicien soit formé à leur repérage et à leur prise en charge.

Conflit d'intérêt : les auteurs déclarent ne pas avoir de conflit d'intérêt. 